\title{
Quantum chaos in a deformable billiard: Applications to quantum dots
}

\author{
Henrik Bruus and A. Douglas Stone \\ Applied Physics, Yale University, \\ P.O.Box 208284, New Haven, Connecticut 06520-8284 \\ (Submitted to Physical Review B15, cond-mat/9406010)
}

\begin{abstract}
We perform a detailed numerical study of energy-level and wavefunction statistics of a deformable quantum billiard focusing on properties relevant to semiconductor quantum dots. We consider the family of Robnik billiards generated by simple conformal maps of the unit disk; the shape of this family of billiards may be varied continuously at fixed area by tuning the parameters of the map. The classical dynamics of these billiards is well-understood and this allows us to study the quantum properties of subfamilies which span the transition from integrability to chaos as well as families at approximately constant degree of chaoticity (Kolmogorov entropy). In the regime of hard chaos we find that the statistical properties of interest are well-described by random-matrix theory and completely insensitive to the particular shape of the dot. However in the nearly-integrable regime non-universal behavior is found. Specifically, the level-width distribution is well-described by the predicted $\chi^{2}$ distribution both in the presence and absence of magnetic flux when the system is fully chaotic; however it departs substantially from this behavior in the mixed regime. The chaotic behavior corroborates the previously predicted behavior of the peak-height distribution for deformed quantum dots. We also investigate the energy-level correlation functions which are found to agree well with the behavior calculated for quasi-zero-dimensional disordered systems.
\end{abstract}

PACS numbers: 73.40.Gk, 05.45.+b, 72.20.My, 73.20.Dx

Typeset using REVTEX 


\section{INTRODUCTION}

The relevance of concepts from the theory of "quantum chaos" to mesoscopic physics has become increasingly clear as nanostructure technology has achieved controlled fabrication of systems smaller than both the elastic and inelastic scattering length $\mathbb{1}$. Quantum chaos is the generally accepted term for properties of a quantum system associated with classical chaos (or the classical transition to chaos). In the most recent high-mobility nanostructures transport is ballistic and the dominant scattering mechanism is the reflection of the electrons at the boundaries of the structure which (depending on the nature of the confining potential) may generate classically chaotic, mixed or integrable dynamics. Thus they present experimental possibilities for the application and testing of concepts from the theory of quantum chaos in condensed matter physics. Disordered mesoscopic systems also are undoubtedly chaotic classically, and recent work has emphasized the similarity between disordered quantum systems and the ballistic systems which have chaotic boundary scattering. In particular at low temperature both type of systems exhibit sample-specific mesoscopic fluctuations in various physical properties as a function of external parameters such as magnetic field. The ballistic systems differ from the disordered systems however in two ways. First, as we will use the term, a disordered system generates elastic scattering of electrons on a scale $l$ which is short compared to typical sample dimensions $L$. This means that transport is diffusive on a scale smaller than the system size and because the diffusion process is dependent on dimensionality the statistical properties in general depend on the spatial dimension. Chaotic ballistic systems on the other hand have no relevant transport length smaller than the system size and thus many of their properties are insensitive to the spatial dimensionality (they are said to be "quasi-zero-dimensional"). It turns out that this difference leads to differences in the statistical properties of disordered and chaotic systems in certain regimes of energy and temperature 23. Second, there is reasonable evidence that ballistic systems may be fabricated with geometries and potential profiles which generate nearly-integrable classical dynamics, thus it becomes worthwhile to consider models which describe the transition to chaos and not just fully chaotic dynamics.

There are two main approaches to the quantum theory of chaotic systems. The approach through semiclassical quantum mechanics pioneered by Gutzwiller $\$$, and the approach based on the theory of random matrices first applied to quantum chaos by Bohigas, Giannoni and Schmit5. The former approach makes a more direct connection to the classical mechanics and has had major successes recently in atomic physics. However the confining potential in the microstructures studied experimentally is rarely well enough known to justify theoretical work relying on specific classical orbits. Instead either a wholly statistical approach, or a combination of semiclassical and statistical ideas has been applied to quantum chaos in mesoscopic systems. Three measured physical effects which have been proposed as manifestations of quantum chaos in mesoscopic transport are: 1) The resistance fluctuations in GaAs quantum wires coupled strongly to an electron cavity 6 6. 2) The weak localization effect in the same system 8. 3) The fluctuations in the Coulomb blockade conductance peaks 10,11 in quantum dots weakly coupled to leads 12 - The electron cavity conductance fluctuations have been described by a combination of statistical and semiclassical theory, and most recently certain properties have been derived from random-matrix theory 16.17 . Whereas the Coulomb blockade peak fluctuations in quantum dots have been described completely 
statistically, using only random-matrix theory. The quantum dot conduction experiments are analogous to strongly resonant scattering in atoms or nuclei for which the properties of a single quasi-bound state can be probed. In micron-size semiconductor quantum dots at the typical experimental density it is estimated that the single-particle level-spacing (or the excitation energy to the first excited state) $\Delta \varepsilon \sim 0.05 \mathrm{meV} \sim 500 \mathrm{mK}$ and therefore these systems may be studied in the regime $k T<\Delta \varepsilon$ where indeed only a single quasi-bound state participates in the resonance.

In this paper we will concentrate on the statistical approach to quantum chaos through random-matrix theory. This theory was originally developed by Wigner, Dyson and others to explain statistical properties of compound nuclear resonances 18,01 but later it was conjectured by Bohigas-Giannoni-Schmit 5 to describe any quantum system whose classical analogue is fully chaotic. There now exists substantial numerical evidence supporting this conjecture as well as an analytic argument due to Berry 20 which applies to a particular statistical property (known as the $\Delta_{3}$ statistic) measuring the long-range rigidity of the spectrum. In RMT complex systems are represented by ensembles of Hamiltonians with statisticallyindependent matrix elements. These ensembles can be shown to have maximum statistical entropy (subject to a small number of constraints)21. There exist three different symmetry classes of such ensembles characterized by the number $\beta=1,2,4$ of independent components of the matrix elements in the Hamiltonian, $H$ : In zero magnetic field without spin-dependent scattering $H$ is real, $\beta=1$, and the corresponding ensemble (invariant under orthogonal transformations) is denoted the Gaussian orthogonal ensemble (GOE). In non-zero magnetic field $H$ is complex, $\beta=2$, and the ensemble (invariant under unitary transformations) is denoted the Gaussian unitary ensemble (GUE). Finally for strong spin-orbit scattering $H$ is quarternion real, $\beta=4$, and the ensemble (invariant under symplectic transformations) is denoted the Gaussian symplectic ensemble (GSE). In this paper we shall neglect spin effects (since spin-orbit scattering is neglibile in the ballistic microstructures) and hence only consider applications of the GOE or GUE.

One well-known property of these statistical ensembles is the appearance of strong levelrepulsion due to the lack of any conserved quantum numbers (other than the energy). This makes the probability density of level spacings, $s$, tend to zero as $s \rightarrow 0$ unlike the Poisson distribution of uncorrelated random variables (which has been shown to describe typical integrable system $\left.{ }^{22}\right)$. Using RMT it is possible to calculate the distribution $P_{\beta}(s)$ in the spectrum normalized such that the local average level spacing is one. For RMT of twodimensional matrices the level spacing distributions are

$$
\begin{aligned}
& P_{1}(s)=\frac{\pi}{2} s \quad \exp \left(-\frac{\pi}{4} s^{2}\right) \quad(\mathrm{GOE}) \\
& P_{2}(s)=\frac{32}{\pi^{2}} s^{2} \exp \left(-\frac{4}{\pi} s^{2}\right) \quad(\mathrm{GUE})
\end{aligned}
$$

Note that this distribution tends to zero as $s^{\beta}$ as $s \rightarrow 0$. Although not exact for $N \times N$ random matrices, these formulae (known as the Wigner surmise) are excellent approximations to the exact results and are conventionally used for comparison to statistical data. The appearance of the RMT spacing distributions at the classical transition to hard chaos has been verified in many numerical studies (although an analytic derivation has still not been found). Hence in this work we will only use the spacing distribution as a diagnostic for the applicability of RMT. 
The theory of these random-matrix ensembles has tended to focus on spectral statistics and not on statistical properties of the eigenstates; however in the application to quantum dot resonances treated below we shall find that it is the eigenstate statistics which are most easily measured. Very recently a great deal of progress has been made in the study of ensembles in which the random-matrix varies as a function of an external parameter such as magnetic flux. Various correlation functions of the spectra have been calculated and shown to be universal upon rescaling23,24. These correlation functions are not easily measured in current experiments on quantum dots, but may be accessible with some effort25. Since such correlation functions are only calculated analytically for random ensembles it is again worthwhile to test their applicability to a given chaotic dynamical system. We perform detailed comparisons of this type below.

Although the applicability of random-matrix theory to quantum systems which are classically chaotic is now reasonably well established, most dynamical systems are neither fully chaotic nor fully integrable, but instead have a mixed classical phase space described topologically by the Kolmogorov-Arnold-Moser (KAM) theorem 26 and related results. The expectation is that RMT will give valid and universal results for fully chaotic hamiltonians and will break down in some manner as regions of stability appear in the phase-space. It is of interest then to look at a model for an ensemble of quantum dots which can span the range from integrability to hard chaos, and can also test universality in the chaotic limit. A model of this type was introduced by Robnik2728 and generalized by Robnik and Berry 29 . In this model the quantum dot is represented by a deformed circular well with infinite walls; the deformation is described by a quadratic or cubic conformal transformation. This model has a number of attractive features for our purposes. 1) The classical mechanics is wellunderstood and has been studied in detail27.30. It has been shown that as the parameters of the conformal transformation are varied the well can undergo a standard KAM transition to chaos. In addition we show that if the parameters are varied in a different manner a sequence of different chaotic billiards are generated with roughly equivalent degrees of classical chaos. Thus one can use this set for statistical averaging. 2) Robnik showed that a very efficient numerical algorithm exists for obtaining a large number of eigenstates and eigenvalues for this model. 3) Berry and Robnik showed that an Aharonov-Bohm flux may be simply introduced into the well to break time-reversal symmetry (without changing the classical dynamics) and that this would only introduce minor changes in the numerical algorithm for solution of the Schrödinger equation. Thus the model is suitable for testing the universality of statistical properties in the chaotic regime and their possible breakdown in the mixed regime for both the orthogonal and unitary ensembles. Preliminary results of this investigation of significantly less breadth have been published elsewhere 4 .

The paper is organized as follows. In Sec. II the deformable billiard is defined in terms of a conformal map. In Sec. III the classical dynamics of the billiard is discussed with the emphasis on characterizing the degree of chaos as a function of deformation. In Sec. IV the quantum mechanics of the billiard is discussed, and the single-electron Schrödinger equation is solved with three different boundary conditions (Dirichlet, Neumann, and general), and the respective level statistics are calculated. In Sec. V using the shape as the external perturbation the energy level correlation functions are calculated for the normalized spectra and comparisons are made with conjectures for their form stated in the literature. In Sec. VI we turn to the problem of fluctuating Coulomb blockade conductance peak heights 
in quantum dots. A model of a Coulomb blockade device is made based on the deformable billiard, and the peak height distributions are calculated and compared to those obtained from random-matrix theory. In Sec. VII some experimental implications are discussed and a new experiment is suggested aimed at testing our results. Finally in Sec. VIII we present a concluding discussion.

\section{DEFINITION OF THE DEFORMABLE BILLIARD}

A mathematically simple way of defining a continuous deformable family of billiards was introduced by Robnik 27 and Berry and Robnik 29 . It is based on a conformal mapping of the unit disk. To make this paper self-contained and to facilitate the presentation of some new developments of the method we shall briefly describe the technique below.

As shown in Fig. 目 we study the simply connected domain $\mathcal{D}$ with a possibly irregular shaped boundary $\partial \mathcal{D}$ in the $u v$ plane. The open interior of the domain is denoted $\mathcal{D} \backslash \partial \mathcal{D}$. The deformed billiard is defined by the infinitely hard wall potential $V(u, v)$ satisfying

$$
V(u, v)=\left\{\begin{array}{cl}
0, & (u, v) \in \mathcal{D} \backslash \partial \mathcal{D} \\
\infty, & (u, v) \notin \mathcal{D} .
\end{array}\right.
$$

Following Robnik 2 we define the shape of $\mathcal{D}$ by a conformal mapping $w$ of the unit disk $\mathcal{C}$ in the $x y$ plane to $\mathcal{D}$ in the $u v$ plane. Parts of the treatment are more conveniently carried out by introducing the complex coordinates $z$ and $w$ :

$$
z \equiv x+i y \quad w=w(z) \equiv u(z)+i v(z) .
$$

The boundary $\partial \mathcal{D}$ in the $w$ plane is thus given as the image $w(\mathcal{C})$ of the boundary of the unit disk $\partial \mathcal{C}$ in the $z$ plane. Throughout this work we study the cubic mapping introduced by Berry and Robnik 29 ,

$$
w(z)=\frac{z+b z^{2}+c e^{i \delta} z^{3}}{\sqrt{1+2 b^{2}+3 c^{2}}}, z \in \mathcal{C},
$$

where $b, c$, and $\delta$ are real parameters chosen such that $\left|w^{\prime}(z)\right|>0$ for $z \in \mathcal{C}$. Two sequences of deformed billiards are show in Fig. 2. This cubic form of $w(z)$ is the simplest conformal map resulting in a billiard with no spatial symmetries (see Fig. 2b). The even simpler quadratic map $(c=0)$ generates a family of billiards with reflection symmetry (see Fig. 2a); Robnik and Berry pointed out that such spatial symmetries can prevent a magnetic flux from generating the orthogonal to unitary transition 3 hence we maintain the more general form although the simple quadratic case is sufficient for many of our calculations. The deformed billiard is given by:

$$
\begin{aligned}
\partial \mathcal{D} & =\{w(z):|z|=1\} \\
\mathcal{D} \backslash \partial \mathcal{D} & =\{w(z):|z|<1\}
\end{aligned}
$$

The normalization in Eq. (5) insures that the area of $\mathcal{D}$ remains $\pi$ for any value of the parameters. 
For later reference we give the explicit form of the Jacobian $\left|w^{\prime}(z)\right|^{2}$ in the polar coordinates $(r, \theta)$ of the $x y$ plane:

$$
\begin{aligned}
\left|w^{\prime}\left(z=r e^{i \theta}\right)\right|^{2}= & {\left[1+4 b^{2} r^{2}+9 c^{2} r^{4}+6 c r^{2} \cos (2 \theta+\delta)\right.} \\
& \left.+4 b r \cos (\theta)+12 b c r^{3} \cos (\theta+\delta)\right] \\
& /\left(1+2 b^{2}+3 c^{2}\right) .
\end{aligned}
$$

\section{THE CLASSICAL DYNAMICS OF THE DEFORMED BILLIARD}

Robnik conducted a thorough study 27 of the classical mechanics of a point mass moving freely in the deformed billiard for the quadratic case $(c=0$, see Fig. 2a). More recently 30 Hayli et al. have extended his results. Thus in contrast to many previous works on ballistic microstructures in which a discretized version of the Schrödinger equation has been studied $22,23,24$ in this case one has a detailed knowledge of the classical dynamics of the relevant quantum system. Robnik showed how as a function of $b$, starting from $b=0$, the system evolves according to the KAM theorem into a mixed phase-space exhibiting soft chaos and eventually to fully developed chaos. The main tool for determining the degree of chaos has been to construct the Poincaré surfaces of section for the bounce map and to calculate the Kolmogorov entropy (we will define these below). The last large islands of stability in the Poincaré section disappear around $b=0.25$ (the value at which the billiard ceases to be convex) and Robnik originally conjectured that the transition to hard chaos occured at this value 27. However recent work has shown that very small islands of stability spawned by the bifurcation of the final stable two-cycle persist up to $b \approx 0.28$, but the precise value of $\mathrm{b}$ at which hard chaos sets in is not known. However it has recently been proven 32 that the quadratic billiard at $b=0.50$ is fully chaotic making this only the third billiard (along with the stadium and Sinai billiards) for which hard chaos has been demonstrated analytically. In practice the islands of stability have negligible weight for $b>0.25$ and the statistical properties of both the classical and quantum mechanics are consistent with fully developed chaos.

The Poincaré section is constructed by plotting the phase space coordinates of the particle each time it reflects specularly from the boundary of the billiard. The Poincaré section is area-preserving if it is constructed using conjugate canonical variables; a useful set of coordinates for billiard $\$ 33$ is the arc length $\sigma$, measured from the origin to the point the particle hits the boundary, and the tangential momentum $\sin (\chi)$ at this point $(\chi$ denotes the angle of incidence). In Fig. 3 we show Poincaré sections corresponding to the deformation sequence of Fig. 2a. For simplicity here we replace $\sigma$, the arc-length, by $\theta$ the angular coordinate. For small deformations these are approximately proportional and since $\sigma$ is a monotonically increasing function of $\theta$ the topology of the surface of section in $\theta$ is the same as in $\sigma$. (strictly speaking our use of $\theta$ introduces some small violation of the area-preserving property which is unimportant since we are only using the SOS for illustrative purposes). The surfaces of section in Fig. 3 clearly show how the deformed billiard gets more chaotic as the deformation is increased from zero. The many large stable islands at the beginning of the sequence shrink and vanish sequentially until one obtains the featureless surface of 
section plot shown in Fig. 3d. In contrast, if one starts from a deformed billiard as in Fig. 2 b and progresses through further deformations, all the surface of section plots look like the featureless plot in Fig. 3 $\mathrm{d}$, indicating that all of these billiards are close to hard chaos.

Quantitative information on the degree of chaos can be obtained by calculating the Lyapunov exponent and the related Kolmogorov entropy2734. Following Benettin 34 the Lyapunov exponent can be estimated numerically by examining two trajectories starting at the phase space points $\boldsymbol{\xi}_{0}^{a}=\left\{\sigma_{0}^{a}, \sin \left(\chi_{0}^{a}\right)\right\}$ and $\boldsymbol{\xi}_{0}^{b}=\left\{\sigma_{0}^{b}, \sin \left(\chi_{0}^{b}\right)\right\}$ separated by a small distance $d_{0}=\left|\boldsymbol{\xi}_{0}^{b}-\boldsymbol{\xi}_{0}^{a}\right| \approx 10^{-10}$. After one bounce the two points $\boldsymbol{\xi}_{1}^{a}$ and $\boldsymbol{\xi}_{1}^{b}$ are reached, the distance $d_{1}$ between them is calculated, and the reduction ratio $\beta_{1}=d_{0} / d_{1}$ is formed. A new rescaled point $\boldsymbol{\xi}_{1}^{b *}$ is defined by $\boldsymbol{\xi}_{1}^{b *} \equiv \boldsymbol{\xi}_{1}^{a}+\beta_{1}\left(\boldsymbol{\xi}_{1}^{b}-\boldsymbol{\xi}_{1}^{a}\right)$. Starting from $\boldsymbol{\xi}_{1}^{a}$ and $\boldsymbol{\xi}_{1}^{b *}$ a second bounce is calculated, and from $\boldsymbol{\xi}_{2}^{a}$ and $\boldsymbol{\xi}_{2}^{b}$ a new reduction ratio $\beta_{2}$ and a new rescaled point $\boldsymbol{\xi}_{2}^{b *}$ is formed and the process is repeated. The Lyapunov exponent $\Lambda$ which generally depends on the initial phase space point can then be calculated as

$$
\Lambda\left(\sigma_{0}^{a}, \sin \left(\chi_{0}^{a}\right)\right)=-\lim _{n \rightarrow \infty}\left(\frac{1}{t_{n}} \sum_{i=1}^{n} \ln \beta_{i}\right),
$$

where $t_{n}$ is the traversal time or the total length in real space of the total $n$-bounce orbit. From the local measure of chaoticity constituted by $\Lambda$, which depends on the initial points in phase space, a global measure the Kolmogorov entropy $h$ can be formed:

$$
h=\frac{1}{2 \mathcal{L}} \int_{-1}^{1} d(\sin (\chi)) \int_{0}^{\mathcal{L}} d \sigma \Lambda(\sigma, \sin (\chi)),
$$

where $\mathcal{L}$ is the perimeter of the billiard and $(\sigma, \sin (\chi))$ is the initial phase space point. The Kolmogorov entropy $h$ provides a quantitative measure of the degree of chaos in the billiard. In Fig. $h$ is shown for the deformation sequences of Fig. 2. Note how $h$ grows monotonically for the sequence (a), while it is fairly constant and significantly bigger for the sequence (b). Thus the Kolmogorov entropy calculations indicate that the deformation sequence Fig. 20 $\mathrm{b}$ is "more chaotic" than the quadratic billiard even at $b=0.50$ where it is known to be fully chaotic. Of course the K-entropy is essentially an average property of the phase-space and does not allow us to exclude some very small regions of stability in the sequence Fig. 2ab.

\section{QUANTUM MECHANICS OF THE DEFORMED BILLIARD}

Following Berry and Robnike one can calculate the quantum states of a single particle moving in the interior of the deformed billiard given by Eq. (6). The billiard is threaded by an Aharonov-Bohm flux tube of strength $\alpha \Phi_{0}$ through the origin of the $u v$ plane. Here $\Phi_{0}$ is the flux quantum $h / e$ and $\alpha$ is a dimensionless real number. Choosing the gauge

$$
\mathbf{A}(u, v)=\frac{\alpha}{2 \pi} \Phi_{0}\left(\frac{\partial f}{\partial v},-\frac{\partial f}{\partial u}, 0\right), \quad f=\frac{1}{2} \ln \left(|z(w)|^{2}\right),
$$

the Schrödinger equation in $w$ coordinates,

$$
\frac{1}{2 m}\left(-i \hbar \nabla_{w}+e \mathbf{A}(w)\right)^{2} \Psi(w)=E \Psi(w),
$$


becomes the following in the polar coordinates $(r, \theta)$ of the $x y$ plane:

$$
\begin{aligned}
& \nabla_{r, \theta}^{2} \Psi(r, \theta)-\frac{i 2 \alpha}{r^{2}} \partial_{\theta} \Psi(r, \theta) \\
& -\frac{\alpha^{2}}{r^{2}} \Psi(r, \theta)+\varepsilon\left|w^{\prime}\left(r e^{i \theta}\right)\right|^{2} \Psi(r, \theta)=0,
\end{aligned}
$$

where now the energy $\varepsilon$ is given in units of $\hbar^{2} / 2 m R^{2}$ and lengths in units of $R, R$ being the radius of the circle $\mathcal{C}$. The spectrum of the Hamiltonian is periodic in $\alpha$ with period 1. Except for the two values $\alpha=0,1 / 2$ for which a real nondiagonal representation of the Hamiltonian can be found the Hamiltonian is a full complex Hermitian operator. Hence GUE statistics is expected for all $\alpha$ except for values close to 0 and $1 / 2$ at which GOE statistics is anticipated. Below whenever we wish to focus on the GUE case we choose the value $\alpha=1 / 4$ to stay as far away as possible from the GOE values.

Thus using the fact that the deformed billiard is obtained by a conformal map of the unit disk we may replace the original problem on the irregular domain $\mathcal{D}$ by an equivalent problem on the unit disk moving under a rather simple "potential" proportional to $\left|w^{\prime}\left(r e^{i \theta}\right)\right|^{2}$. In the simplest cases of Dirichlet or Neumann boundary conditions discussed in sections A and $\mathrm{B}$ below the boundary conditions of the equivalent problem are identical to those on the original domain. Since a convenient basis which satisfies these boundary conditions is available (products of Bessel functions, $J_{\nu}(\gamma r)$, and exponentials, $\exp (i l \theta)$ ) Eq. (12) may be expressed as a linear equation in that (infinite) basis and after truncation may be solved numerically. Typically in this study we truncate Eq. (12) to the subspace of the 1000 lowest energy Bessel solutions. As noted by Robnik, the dependence of this linear equation on the shape parameters $b, c$, and $\delta$ can be factored out allowing a very efficient numerical method of solution as the shape is varied. To discuss physical properties relating to isolated quantum dots it is reasonable to consider this model with Dirichlet boundary conditions; however we are also interested in fluctuation properties related to conductance. In this case the deformed dot must be coupled to leads (typically through tunnel barriers) and more general boundary conditions must be imposed to describe its scattering resonances. Hence in the following subsections we discuss the solution of Eq. (12) under Dirichlet, Neumann and finally general inhomogeneous boundary conditions.

\section{A. Dirichlet boundary conditions (DBC)}

Eq. (12) with the Dirichlet boundary conditions (DBC)

$$
\Psi(w)=0, \text { for } w \in \partial \mathcal{D} .
$$

was considered in the original work of Berry and Robnik 29 . As just noted, a simplifying property of the conformal mapping is that if $\Psi(w)$ satisfy $\mathrm{DBC}$ on the domain $\mathcal{D}$, so will $\Psi(w(z))$ on the circle $\mathcal{C}$. We therefore expand the solutions of the Schrödinger equation (12) with DBC in terms of the eigenstates $\left|\phi_{l, n}\right\rangle$ of the analogous Schrödinger equation for the unit disk $\mathcal{C}$ also with DBC. These are given by

$$
\left\langle r, \theta \mid \phi_{l, n}\right\rangle=\frac{1}{\sqrt{\pi} J_{|l-\alpha|}^{\prime}\left(\gamma_{|l-\alpha|, n}\right)} J_{|l-\alpha|}\left(\gamma_{|l-\alpha|, n} r\right) e^{i l \theta}
$$


where $r \leq 1, J_{\nu}$ is the Bessel function of order $\nu, \gamma_{\nu, n}$ is the $n$th root of $J_{\nu}$, and $l$ is an integer. The eigenvalues of $\left|\phi_{l, n}\right\rangle$ are given by

$$
-\nabla^{2}\left|\phi_{l, n}\right\rangle=\gamma_{|l-\alpha|}^{2}\left|\phi_{l, n}\right\rangle \text {. }
$$

Note that degeneracies occur in the energy spectrum in case of zero AB-flux $(\alpha=0)$.

It is convenient to enumerate the basis states not according to the quantum numbers $(l, n)$ but according to the position $j$ of the eigenvalues $\gamma^{2}$ arranged in ascending order. The eigenstates $\psi_{p}$ of the Schrödinger equation in the deformed billiard with DBC are now written as the superposition

$$
\left|\psi_{p}\right\rangle=N_{p} \sum_{j=1}^{\infty} \frac{c_{j}^{(p)}}{\gamma_{j}}\left|\phi_{j}\right\rangle,
$$

where $N_{p}$ is a normalization constant. The particular form of the expansion coefficients is chosen so that the transformed Schrödinger equation (12) becomes a Hermitian eigenvalue problem. The coefficients $c^{(p)}$ and the eigenvalues $\varepsilon_{p}$ are found from Eq. (12) by insertion of the superposition Eq. (16) followed by multiplication with $\left\langle r, \theta \mid \phi_{i}\right\rangle$ and integration over $r$ and $\theta$. After rearrangement the eigenvalue problem then takes the form

$$
M_{i j} c_{j}^{(p)}=\frac{1}{\varepsilon_{p}} c_{j}^{(p)}
$$

The explicit form of the Hermitian matrix $M$ using Eq. (]) for the Jacobian is the following:

$$
\begin{aligned}
M_{i j}=\left[\delta_{i j}\right. & +\delta_{l_{i}, l_{j-2}} 6 c e^{-i \delta} I_{i j}^{(2)} \\
& +\delta_{l_{i}, l_{j-1}}\left(4 b I_{i j}^{(1)}+12 b c e^{-i \delta} I_{i j}^{(3)}\right) \\
& +\delta_{l_{i}, l_{j+0}}\left(8 b^{2} I_{i j}^{(2)}+18 c^{2} I_{i j}^{(4)}\right) \\
& +\delta_{l_{i}, l_{j+1}}\left(4 b I_{i j}^{(1)}+12 b c e^{+i \delta} I_{i j}^{(3)}\right) \\
& \left.+\delta_{l_{i}, l_{j+2}} 6 c e^{+i \delta} I_{i j}^{(2)}\right] /\left(1+2 b^{2}+3 c^{2}\right),
\end{aligned}
$$

where the integrals $I_{i j}^{(h)}$ are given by

$$
I_{i j}^{(h)}=\frac{\int_{0}^{1} d r r^{h+1} J_{\nu_{i}}\left(\gamma_{i} r\right) J_{\nu_{j}}\left(\gamma_{j} r\right)}{\gamma_{i} \gamma_{j} J_{\nu_{i}}^{\prime}\left(\gamma_{i}\right) J_{\nu_{j}}^{\prime}\left(\gamma_{j}\right)}
$$

with $\nu_{i}=\left|l_{i}-\alpha\right|$. For given values of the parameters $b, c$, and $\delta$ we construct the truncated $M$-matrix for the 1000 lowest eigenstates $\left|\phi_{j}\right\rangle$. The 300 highest eigenvectors of $M$ corresponding to the 300 lowest eigenstates of our original Hamiltonian are accurate enough for our analysis. Typically the eigenvalues $\varepsilon_{p}$ are more accurate than 0.001 times the average level spacing, and among the 300 levels only a few of the highest ones, where the presence of the matrix truncation is felt most strongly, have an accuracy as low as around 0.1 times the average level spacing.

Once the energy spectrum is obtained for the lowest 300 eigenstates various level statistics can be calculated and compared with the predictions of RMT. For specific shapes (parameter 
values) this has been done previously by Berry and Robnik ${ }^{29}$ and later by Goldberg et al. . $^{2}$, finding excellent agreement with e.g. the spacing distribution predicted by RMT in the chaotic regime. Since we will use a range of shapes not covered by these studies we confirm the expectation that these results extend to the wider class of chaotic billiards studied below.

The first step in the energy level analysis is to "unfold" the spectrum. This procedure corrects for the average variation in the density of states with energy so that the mean levelspacing of the unfolded spectrum is always unity and data in different energy intervals may be merged. For a smooth billiard obeying DBC the Weyl formula states that the average number of states with energy less than $\varepsilon, N(\varepsilon)$, is given (for high energies) by 36 :

$$
N(\varepsilon)=\frac{1}{4} \varepsilon-\frac{\mathcal{L}}{4 \pi} \sqrt{\varepsilon}+\frac{1}{6},
$$

where $\mathcal{L}$ is the perimeter of the billiard, which we calculate by numerical integration. The unfolded eigenenergy corresponding to the eigenenergy $\varepsilon_{j}$ is given by $N\left(\varepsilon_{j}\right)$, and the $j$ th unfolded level spacing $s_{j}$ is thus

$$
s_{j} \equiv N\left(\varepsilon_{j+1}\right)-N\left(\varepsilon_{j}\right) .
$$

After unfolding the calculated energy spectra a histogram for the distribution of the level spacings can be constructed, an example of which is shown in Fig. 5. The hypothesis that the histograms are following the GOE distribution of Eq. (11) or the GUE distribution of Eq. (2) can now be tested statistically. The quantitative measure we use is the one-sided

$\chi^{2}$-test, which estimates the probability $P\left(\chi^{2}\right)$ for finding a $\chi^{2}$-value bigger than the actual observed one.

In Fig. (6) we show the results of the $\chi^{2}$-test for the GOE and GUE distributions. We have calculated the energy spectra for 78 different values of $\delta$ in the interval $[0, \pi]$ for constant $b$ and $c$, both being equal to 0.2 (the deformation sequence shown in Fig. $2 \mathrm{~b}$ ). The billiard was threaded by an AB-flux tube of strength $\frac{1}{4}$ of a flux quantum. It is seen, as expected from Ref. 31, that the level distribution with statistical significance is given by the GUE distribution in most of the parameter range. However, we also note that for $\delta \approx 0$ and $\delta \approx \pi$ where the billiard is almost symmetrical around the $u$-axis, the GOE distribution better describes the spectrum. When comparing the results for the classical mechanics in Sec. III with those obtained here for the quantum mechanics, we obtain yet another verification of the observation that the energy level distribution of a quantum system whose classical counterpart exhibits hard chaos follows Wigner's distribution. Although this result was expected, we note that our study provides a more complete test of the validity of this statement than previous studies in that we test a range of billiards with roughly constant Kolmogorov entropy.

\section{B. Neumann boundary conditions (NBC)}

A central issue in this paper is to study not completely isolated but nearly isolated systems where the electrons can escape. Therefore we have to abandon the DBC of Eq. (13) which are relevant only for closed systems. As we will discuss later, a partially open system will require not just boundary conditions but also matching conditions for solutions inside 
and outside. Such matching conditions can be expressed in terms of any basis set for the region $\mathcal{D}$ which does not cause the wavefunction to vanish identically (as do DBC). The mathematically simplest alternative choice is Neumann boundary conditions (NBC) for which the normal derivative vanishes everywhere on the boundary:

$$
\mathbf{n} \cdot \nabla \Psi(w)=0, \quad w \in \partial \mathcal{D},
$$

where $\mathbf{n}$ is the outward pointing normal of the boundary at the point $w$.

Although not treated in previous work NBC maintain the simplicity of DBC in that they are preserved by the conformal map, i.e. if $\Psi(w)$ obeys Eq. (22) so will $\Psi(w(z))$ for $z \in \partial \mathcal{C}$, and there exists a simply basis for the unit disk satisfying NBC. We can therefore solve the Schrödinger equation Eq. (12) with NBC following the steps in Sec. IV-A. The only modification is a change of the radial part of the basis functions defined in $\mathcal{C}$ as they appear in Eq. (14) to the following (where a tilde is used to distinguish NBC from DBC):

$$
\left\langle r, \theta \mid \tilde{\phi}_{j}\right\rangle=\frac{\tilde{\gamma}_{j} / \sqrt{\pi}}{\sqrt{\tilde{\gamma}_{j}^{2}-\left|l_{j}-\alpha\right|^{2}} J_{\left|l_{j}-\alpha\right|}\left(\tilde{\gamma}_{j}\right)} J_{\left|l_{j}-\alpha\right|}\left(\tilde{\gamma}_{j} r\right) e^{i l_{j} \theta} .
$$

We have again ordered the basis set in ascending order according to the eigenvalues $\tilde{\gamma}_{j}^{2}$, with $\tilde{\gamma}_{j}$ now being a root of the derivative $J_{\nu_{j}}^{\prime}$ of the Bessel function $J_{\nu_{j}}$. The solution $\left|\tilde{\phi}_{p}\right\rangle$ of Schrödinger equation with NBC in the deformed billiard is written in analogy with Eq. (16) as:

$$
\left|\tilde{\psi}_{p}\right\rangle=\tilde{N}_{p} \sum_{j=1}^{\infty} \frac{\tilde{c}_{j}^{(p)}}{\tilde{\gamma}_{j}}\left|\tilde{\phi}_{j}\right\rangle
$$

The matrix equation (17) becomes

$$
\tilde{M}_{i j} \tilde{c}_{j}^{(p)}=\frac{1}{\tilde{\varepsilon}_{p}} \tilde{c}_{j}^{(p)}
$$

The explicit form of the Hermitian matrix $\tilde{M}$ is obtained from Eq. (18) by substituting the integrals $I_{i j}^{(h)}$ with the integrals $\tilde{I}_{i j}^{(h)}$ given by

$$
\tilde{I}_{i j}^{(h)}=\frac{\int_{0}^{1} d r r^{h+1} J_{\nu_{i}}\left(\tilde{\gamma}_{i} r\right) J_{\nu_{j}}\left(\tilde{\gamma}_{j} r\right)}{\sqrt{\tilde{\gamma}_{i}^{2}-\nu_{i}^{2}} \sqrt{\tilde{\gamma}_{j}^{2}-\nu_{j}^{2}} J_{\nu_{i}}^{\prime}\left(\tilde{\gamma}_{i}\right) J_{\nu_{j}}^{\prime}\left(\tilde{\gamma}_{j}\right)},
$$

where $\nu_{i}=\left|l_{i}-\alpha\right|$. The energy level statistics under NBC can now be studied by calculating the normalized energy spectrum using numerical diagonalization of the truncated 1000 by 1000 sub-matrix of $\tilde{M}$. The Weyl formula for the NBC case is 36

$$
N(\tilde{\varepsilon})=\frac{1}{4} \tilde{\varepsilon}+\frac{\mathcal{L}}{4 \pi} \sqrt{\tilde{\varepsilon}}+\frac{1}{6},
$$

where in comparison with the DBC case Eq. (20) the only change is the sign of the second term on the right-hand side. 
Although the change from DBC to NBC leads to relatively minor changes in the method of solution, the relation between the quantum mechanics and the classical mechanics studied in Sec. III becomes less clear since it is natural to associate the hard walls in the classical problem with DBC in the quantum problem. Thus it is of some interest to see if the relation between classical chaos and RMT is independent of this change in the boundary conditions of the quantum problem. The histograms of the level spacing distributions for the NBC case are calculated for the same AB-flux and the same shape deformation sequence as for the DBC case, and $\chi^{2}$-tests for the GOE and GUE level statistics are performed. The result is shown in Fig. 17, where it is seen that again the level spacing distributions are well described by GUE statistics, except near the special values of $\alpha=0,1 / 2$. We observe that the crossover region between GOE and GUE statistics near the $\delta=0$ is wider in the NBC case than the DBC case. We have no physical explanation for this behavior and suspect it is a numerical artifact. Nonetheless the results overall indicate that the correspondence between classical chaos and RMT behavior for the spacing distribution is independent of the boundary conditions.

\section{General boundary conditions (GBC)}

As noted in our introduction, one proposed application of RMT to quantum dots relates to the peak amplitude fluctuations in resonant tunneling through the dot. In such a case electrons may enter and leave the dot in certain directions through leads separated from the dot by tunnel barriers. Thus the problem of interest has the geometry illustrated in Fig. 8. The Schrödinger equation now has solutions at all energies although resonances will still occur at an energy-spacing comparable to that of the closed system. For such a geometry the physically relevant boundary conditions are Dirichlet on the portion of the billiard unconnected to the leads (we denote this part of the boundary $\partial \mathcal{D}_{1}$ ) combined with matching conditions at the leads. In general one may expand the wavefunction inside the dot in any basis which allows the matching procedure at the leads, (i.e. such that the sum over basis functions is uniformly convergent). Unfortunately Dirichlet boundary conditions at the leads do not satisfy this condition, thus one is forced to use a basis which satisfies mixed boundary conditions on $\partial \mathcal{D}$; DBC on $\partial \mathcal{D}_{1}$ and general inhomogenous boundary conditions on the complement $\partial \mathcal{D}_{2}$.

$$
\begin{aligned}
g(w) \mathbf{n} \cdot \nabla \Psi(w)+\Psi(w) & =0, \quad w \in \partial \mathcal{D} \\
g(w) & =0, \quad w \in \partial \mathcal{D}_{1} \\
g(w) & \neq 0, \quad w \in \partial \mathcal{D}_{2}
\end{aligned}
$$

We refer to these as general boundary conditions, GBC. Unlike DBC and NBC, GBC are changed by the conformal mapping; in fact the function $g(w)$ will transform to $g(w(z)) /\left|w^{\prime}(z)\right|$. However a more fundamental problem is that no simple basis set exists on the unit disk which satisfies GBC, so no straightforward extension of the DBC and NBC approach is possible. Of course the problem we are encountering occurs in other contexts (e.g. electromagnetic waveguides) and with significantly more effort we are able to use existing techniques to get an approximate solution. We employ a two-step procedure. First we 
find the eigenstates of the deformed billiard obeying DBC, and then we use the method developed by Feshbach 37 to perturb the $\mathrm{DBC}$ at $\partial \mathcal{D}_{2}$ with the GBC of Eq. (28). The strength of the perturbation is given by the function $g(w)$. If $g(w)$ were zero everywhere along $\mathcal{D}$ we recover DBC, and if $g(w)$ were infinite everywhere along $\mathcal{D}$ we get NBC. The method we use below only applies in the case of zero magnetic field.

Our point of departure is the eigenstates $\left|\psi_{p}\right\rangle$ of Eq. (16) obeying DBC. We have

$$
\nabla^{2} \psi_{p}(w)+\varepsilon_{p} \psi_{p}(w)=0 \quad \psi_{p}(S)=0
$$

where $S$ here and in the following denotes a point on the boundary $\partial \mathcal{D}$. Our aim is to find a solution $X_{\lambda}$ in $\mathcal{D}$ satisfying GBC:

$$
\begin{aligned}
\nabla^{2} X_{\lambda}(w)+E_{\lambda} X_{\lambda}(w) & =0 \\
g(S) \mathbf{n} \cdot \nabla X_{\lambda}(S)+X_{\lambda}(S) & =0 .
\end{aligned}
$$

We begin with the Green's function $G_{\lambda}\left(w \mid w_{0}\right)$ which satisfies DBC:

$$
\begin{aligned}
\nabla_{0}^{2} G_{\lambda}\left(w \mid w_{0}\right)+E_{\lambda} G_{\lambda}\left(w \mid w_{0}\right) & =-\delta\left(w-w_{0}\right) \\
G_{\lambda}(w \mid S) & =0 .
\end{aligned}
$$

Eqs. (30) and (31) together with the use of Green's theorem yields

$$
\begin{aligned}
X_{\lambda}(w) & =-\oint_{\partial \mathcal{D}} d S \mathbf{n} \cdot \nabla_{0} G_{\lambda}(w \mid S) X_{\lambda}(S) \\
& =+\oint_{\partial \mathcal{D}} d S \mathbf{n} \cdot \nabla_{0} G(w \mid S) g(S) \mathbf{n} \cdot \nabla X_{\lambda}(S) .
\end{aligned}
$$

Taking the gradient $\nabla_{w}$ of Eq. (32) and expanding $\nabla X_{\lambda}$ in terms of $\nabla \psi_{p}$,

$$
\nabla X_{\lambda}=\sum_{p} \frac{d_{p}^{(\lambda)}}{\varepsilon_{p}} \nabla \psi_{p}
$$

one obtains after some analysis 37 the following eigenvalue problem involving the expansion coefficients $d_{p}^{(\lambda)}$ and the eigenvalues $E_{\lambda}$ :

$$
\sum_{q}\left\{\left[\frac{1}{\varepsilon_{p}} \delta_{p, q}+\frac{1}{\varepsilon_{p} \varepsilon_{q}} \oint_{\partial \mathcal{D}} d S \mathbf{n} \cdot \nabla \psi_{p}^{*}(S) g(S) \mathbf{n} \cdot \nabla \psi_{q}(S)\right]-\frac{1}{E_{\lambda}} \delta_{p, q}\right\} d_{q}^{(\lambda)}=0 .
$$

Since we are expanding the wave function $X_{\lambda}$ obeying GBC in a basis set $\left\{\psi_{p}\right\}$ obeying DBC some discussion of convergence properties is necessary. The expansion in Eq. (33) converges only point-wise, and the convergence is slowest near the non-DBC part $\partial \mathcal{D}_{2}$ of the boundary. There one might expect the occurence of "overshooting" (the Gibbs phenomena) of the summed series. However, the convergence is good in a least-squares sense, meaning that quantities involving integration over the whole domain $\mathcal{D}$ converge well if the period of the smallest oscillation in the series is much smaller than the extension of the region where $g(S)$ is non-zero. For semiclassical concepts to be relevant we require that the leads be many wavelengths across, but we also wish them to be small compared to the radius of 
the dot. We chose to use leads with a width of about $\pi / 6$ corresponding to about five times the smallest azimuthal wave length when the basis is truncated between 700 and 1000. The energies $E_{\lambda}$, which is essentially the average of $\nabla^{2} X_{\lambda}$ over $\mathcal{D}$ is an example of a quantity with a good least-square convergence. This convergence improves the smaller the strength for $g(S)$. The DBC wavefunctions $\psi_{p}$ appearing in Eq. (33) are only known in terms of the Bessel solutions of Eq. (16). It is thus convenient to map the line integral in Eq. (34) back to the unit disk where it becomes a sum of integrals along the perimeter of the unit disk with $\psi_{p}$ and $\psi_{q}$ replaced by Bessel functions. This mapping introduces an additional factor in the integrand $\left|w^{\prime}(z)\right|^{-1}$. A neat simplification can then be achieved by choosing the function $g(S)$ as

$$
g(S) \equiv g\left(e^{i \theta}\right) \equiv\left\{\begin{array}{cl}
0.1\left|w^{\prime}\left(e^{i \theta}\right)\right|, & , \theta \in[0.5,1.0] \cup[4.5,5.0] \\
0 & , \theta \notin[0.5,1.0] \cup[4.5,5.0]
\end{array}\right.
$$

This choice cancels the factor $\left|w^{\prime}(z)\right|^{-1}$ mentioned above and allows simple analytical evaluation of the line integrals. This greatly improves the numerical tractability of the calculation. The strength of the perturbation $g(S)$ is chosen so that the perturbation is strong enough to shift the individual levels of the order one mean level spacing and such that it is weak enough to avoid substantial mixing of the DBC-eigenstates invalidating the truncation we make at the second step of the procedure. In the following we show the result of calculating the energy spectrum in the GBC case based on the 700 lowest eigenstates of the DBC case.

Once we have obtained the spectrum unfolding it requires a knowledge of the relevant Weyl formula since the subleading corrections depend on the choice of boundary conditions. Unfortunately there does not exist an analytical expression analogous to Eqs. (20) and (27) for GBC. However, the general theory 36 implies that $N(\varepsilon)$ must have the form

$$
N(\varepsilon)=\frac{1}{4} \varepsilon+B \sqrt{\varepsilon}+C .
$$

The constants $B$ and $C$ relating to the boundary and the curvature of the billiard can then be estimated by numerical fitting for each spectrum in question. We tested this procedure on the DBC and NBC spectra and obtained the known coefficients with only a few percent error.

In Fig. 9 we show a comparison of $\chi^{2}$ tests of the numerical spacing distribution calculated with GBC compared to the Wigner surmise for the GOE and GUE for a range of spectra in the deformation sequence shown in Fig. 2 $\mathrm{b}$ for zero AB flux. We see that GOE statistics is well-supported by the data. Taken together the results of sections A,B and C strongly support the conjecture that the correspondence between RMT spectral statistics and classical chaos holds independent of the boundary conditions on the quantum problem.

\section{PARAMETRIC ENERGY LEVEL CORRELATIONS}

Recently a great deal of theoretical work on disordered systems and RMT has examined the statistical correlation of energy levels when the hamiltonian varies as a function of some

parameter such as flux 23:24.35. The analytic results obtained apply to disordered systems 
or to RMT ensembles not generated from a microscopic hamiltonian. Some nice numerical confirmation of the results has been obtained for ordered but irregular systems by Szafer and Altshuler 23 but not for models in which the classical mechanics was known. For example no numerical or analytic results are available for such correlations in the mixed regime. In this section we examine such correlations using the deformation parameters $b$ and $\delta$ as the control variable. We use the closed billiard with the simple DBC discussed in Sec. IV-A, and the deformation sequences shown in Fig. 2. In Fig. 2a only $b$ is changed while in Fig. 2b only $\delta$ varies, hence $b$ and $\delta$ will be the control variables relevant to each sequence, which we will denote generically as $X$. For a given sequence of parameter values the normalized energy spectrum is calculated. The resulting dimensionless energy levels are denoted $\varepsilon_{i}(X)$. The prediction is that for fully chaotic systems certain correlation functions of the $\varepsilon_{i}(X)$ are universal upon rescaling of $X$. Following Ref. 24 we define the generalized conductance $C(0)$ and the rescaled parameter $x$ as

$$
C(0) \equiv\left\langle\left(\frac{\partial \varepsilon_{i}(X)}{\partial X}\right)^{2}\right\rangle \quad x \equiv \sqrt{C(0)} X
$$

where $\langle\cdots\rangle$ is a statistical average over a suitable range of energy and/or $X$. The normalized energy level correlation function $c(x)$ defined as:

$$
c(x) \equiv\left\langle\frac{\partial \varepsilon_{i}(\bar{x}+x)}{\partial \bar{x}} \frac{\partial \varepsilon_{i}(\bar{x})}{\partial \bar{x}}\right\rangle
$$

is predicted to be a universal function which differs for GOE and GUE.

The correlation function is calculated for the deformation sequence shown in Fig. 2 $\mathrm{b}$ with the parameter $\delta$ restricted to the interval $[0.18,2.98]$ to avoid the symmetry points at 0 and $\pi$. The billiard is threaded by one quarter of a flux quantum and DBC is employed. The resulting normalized spectrum for the 50 levels between level number 225 and 274 is shown in Fig. 10. Since the spectrum changes both as a function of energy and as a function of $\delta$ the spectrum has been divided into 5 by 8 boxes each containing 25 energy levels at 14 different values of $\delta$. Within each box the spectrum is fairly homogeneous, and the averaging of Eqs. (37) and (38) is performed within each box. For each box 14 values of the pair $\{x, c(x)\}$ can thus be calculated - the value of $\{0, c(0)\}$, however, is trivially $\{0,1\}$. The resulting 560 points $\{x, c(x)\}$ are then arranged in ascending order after their first component, they are grouped in 56 groups of ten, and the average value of each group is calculated. The final points are displayed in Fig. 11]a. The same procedure is repeated for the case with zero AB-flux and the resulting points are shown in the same figure.

Fig. 11 a shows an excellent agreement between the predicted curves calculated in Refs. 23 and 24 for both the GOE and GUE cases. Thus our results support the conjecture that these correlations are universal and occur when the quantum system is classically chaotic. We can go further however and test whether these correlations actually coincide with classical chaos by looking at $c(x)$ in the mixed regime. Our results shown in Fig. 11b provide support for this stronger statement. $c(x)$ in the mixed regime deviates strongly from its behavior for hard chaos. In particular a large dip appears in the correlation function which can be traced to the average distance to the first level anti-crossing. Unlike the chaotic regime where anticrossings have large gaps, very small gap anti-crossings occur in the mixed regime leading 
to a large parametric derivative of the energy levels. In fact it is known 28 that at such a anti-crossing in the mixed regime two wavefunctions "exchange identities" with the higher energy wavefunction having a spatial density close the the lower-energy wavefunction which has just been repelled. Thus we find that the spectral correlations are non-universal and inconsistent with RMT in the mixed regime.

\section{STATISTICS OF COULOMB BLOCKADE CONDUCTION PEAKS}

As noted above, fluctuations in the spectrum of quantum dots are not yet easily accessible experimentally. The most striking fluctuation effect evident in the experimental data $10,11,14$ are fluctuations in the peak height of Coulomb blockade resonances. These fluctuations reflect properties of the quasi-bound states(level-width fluctuations) and not of the spectrum. This contrasts with nuclear scattering resonances in which both spectral and level-width fluctuations are equally accessible. The reason for this difference 12,14 is that the quantum dot resonances correspond to the ground state energy of the system with $N, N+1, N+$ $2, \ldots$ electrons and thus include the charging energy $e^{2} / C$ associated with the addition of a particle. Since this charging energy is approximately constant and is typically an order of magnitude larger than the single-particle excitation energy, $\Delta \varepsilon$ (or more precisely the energy to the first excited state for fixed $N$ ) the observed resonances are equally-spaced to a good approximation. In addition typically $k T>>\bar{\Gamma}$ (the mean level-width at zero temperature) so the resonances are thermally-broadend to a width $\sim k T$ and only their amplitude reflects the level-width fluctuations. The amplitude fluctuations become maximal when $k T<\Delta \varepsilon$ and only the ground-state contributes to the resonance. In recent experiment.10 $14 \Delta \varepsilon \sim 0.5 \mathrm{~K}$ so that this single-level regime is accessible. The cross-over between multiple-level and singlelevel tunneling leads to unusual and fluctuating temperature-dependences for the resonances until $k T \ll \Delta \varepsilon$, as was first understood by Meir et al.38. In earlier work 12214 we have developed a detailed theory of the amplitude fluctuations in the single-level regime assuming that RMT describes the quasi-bound eigenstate fluctuations. Numerical tests of the theory agreed well for the GOE case but not as well for the GUE case 12 , and were performed for a disordered model which was assumed to generate chaotic classical dynamics. Here we extend and improve these numerical tests by using the conformal billiard model treated above.

As before we model the quantum dot as a deformed billiard accessible by tunneling from leads as shown in Fig. 8. We neglect electron-electron interactions for the following reasons. First they will add a charging energy which is irrelevant to the level-width fluctuations. Second, although the quasi-bound levels in the presence of electron-electron interactions will surely differ from those in its absence, we do not expect this difference to change their statistical properties (at least in the chaotic case). This concept underlies the universality of RMT and is supported by experimental and theoretical work in nuclear scattering. For example complicated shell-model calculations including the residual nuclear interaction lead to spectra which exhibit RMT statistic\$19. The fact that RMT statistics arise in disordered or chaotic non-interacting quantum hamiltonians by no means imply that they are occur only when interactions are negligible.

As in nuclear physics 39 one may relate the scattering resonances to the eigenstates $X_{\lambda}(u, v)$ of the dot in isolation using R-matrix theory 12 14. In the standard approach to 
elastic scattering from nuclei (for which spherical symmetry may be assumed) a linear relationship is derived in each angular momentum channel between the scattering wave function and its derivative at the interface between the nucleus and free space. The coefficient of proportionality is denoted by $R(E)$. If $M$ different decay channels exist this linear relationship defines a matrix of coefficients known as the R-matrix for the nuclear reaction. In our case we do not have spherical symmetry so an angular momentum expansion is inappropriate, but we have a simplification due to the fact that only $M$ propagating modes exist at the Fermi energy in each of the two leads. Moreover, in general the tunneling rate (barrier penetration factor) will be largest for tunneling into the lowest propagating mode, so we can in first approximation neglect all but this mode in each lead. With this approximation and taking the simplest case of Neumann boundary conditions for the $X_{\lambda}$ a derivation very similar to that used in the nuclear case yields 13 a $2 \times 2 R$-matrix of the form

$$
R_{m n}(E)=-\sum_{\lambda=1}^{\infty} \frac{\gamma_{\lambda}^{m} \gamma_{\lambda}^{n}}{E-E_{\lambda}}
$$

where now the index $m(=r, l)$ simply denotes the left or right leads, and the quantities $\gamma_{\lambda}^{m}$ and $E_{\lambda}$ are determined by the solutions of the Schrödinger equation Eq. (30).

$$
\gamma_{\lambda}^{m}=\sqrt{P_{\lambda}} \int_{-W / 2}^{+W / 2} d \eta_{m} Y\left(\eta_{m}\right) X_{\lambda}\left(\xi_{m}^{0}, \eta_{m}\right),
$$

where $P_{\lambda}$ is the barrier penetration factor, $\xi_{m}$ denotes the direction parallel to lead $m$ and $\eta$ that perpendicular, $Y\left(\eta_{m}\right)$ is the transverse wave function in the leads (which have width $W$ ), and $\xi_{m}^{0}$ is the position of the inner edge of tunnel barrier $m$. In deriving this expression we assumed that the tunnel barrier is approximately uniform in the transverse direction. If we were trying to derive information about specific resonances of this system from the Rmatrix then we would need to use a more general R-matrix than Eq. (39) derived for the GBC relevant to our problem. However we are only interested in statistical properties of the Rmatrix and our results above indicate strongly that the statistical properties are independent of the boundary conditions imposed to a good approximation (as long as they allow nonvanishing wavefunctions at the tunnel barriers). Therefore we use the more convenient NBC for the calculations below.

An exact non-linear relationship exists between the S-matrix and the R-matrix ${ }^{13}$; however a particularly useful feature of this formulation is that as $E$ approaches a particular $E_{\lambda}$, the term in Eq. (39) containing the corresponding $X_{\lambda}$ will dominate (as long as coupling to the leads is weak) and all other terms may be neglected. In this approximation the relationship between the $S$ and $R$ matrices simplifies to yield the Breit-Wigner formula for the resonance line-shape under very general conditions. Moreover the level-width which appears in this expression is simply

$$
\Gamma_{\lambda}^{l, r}=\frac{\hbar^{2} k}{m}\left|\gamma_{\lambda}^{l, r}\right|^{2} \equiv \frac{\hbar^{2} k}{m} P_{\lambda}^{l, r}\left|\tilde{\gamma}_{\lambda}^{l, r}\right|^{2},
$$

where we have defined the reduced width $\tilde{\gamma}_{\lambda}$. This reduced width will fluctuate from level to level (and for different lead positions for a given level) due to the complicated spatial structure of the chaotic eigenfunctions $X_{\lambda}$ (see Fig. 8). For example, if there happens to be 
a nodal line near the position of a given lead then the width associated with that level and that lead will fluctuate down. (Note that in the absence of spatial symmetry another lead attached a few wavelengths away will give a completely uncorrelated width for the same level).

In order to relate the level-widths to the experimentally-observed peak amplitudes we use the expression for the peak height $g_{\max }$ derived by Beenakker 0 for the single-level regime (assuming $k T \gg \bar{\Gamma}$ ),

$$
g_{\max }=\frac{e^{2} / h}{4 \pi k T} \frac{\Gamma_{\lambda}^{l} \Gamma_{\lambda}^{r}}{\left(\Gamma_{\lambda}^{l}+\Gamma_{\lambda}^{r}\right)}=\frac{e^{2}}{4 \pi h} \frac{\bar{\Gamma}}{k T} \alpha_{\lambda}
$$

where $\Gamma_{\lambda}=\Gamma_{\lambda}^{l}+\Gamma_{\lambda}^{r}$ is the total decay width for level $\lambda$ and $\Gamma_{\lambda}^{l}, \Gamma_{\lambda}^{r}$ are the partial decay widths into the right and left leads. The factor $\alpha_{\lambda}$ in Eq. (42) is a dimensionless measure of the area under the $T=0 \lambda$-resonance, hence the observed amplitude fluctuations reflect the fluctuations in these areas. In this discussion we will only treat the case of equal barriers on each side of the dot and hence $P_{\lambda}(E)$ will be the same on the left and right. This means that the average decay widths to the left and right are equal, $\bar{\Gamma}^{l}=\bar{\Gamma}^{r}=\bar{\Gamma} / 2$ and we can express $\alpha_{\lambda}$ as

$$
\alpha_{\lambda}=\frac{\Gamma_{\lambda}^{l} \Gamma_{\lambda}^{r}}{\bar{\Gamma}\left(\Gamma_{\lambda}^{l}+\Gamma_{\lambda}^{r}\right)}=\frac{\left|\tilde{\gamma}_{\lambda}^{l}\right|^{2}\left|\tilde{\gamma}_{\lambda}^{r}\right|^{2}}{\left(\left|\tilde{\gamma}_{\lambda}^{l}\right|^{2}+\left|\tilde{\gamma}_{\lambda}^{r}\right|^{2}\right)} .
$$

The statistics of the peak amplitude fluctuations then follow from those of the reduced partial widths $\left|\tilde{\gamma}_{\lambda}\right|^{2}$ using Eqs. (42) and (43).

If we assume that the resonance wave functions $X_{\lambda}$ are described by the GOE when time-reversal symmetry is present $(B=0)$ and by the GUE when time-reversal symmetry is broken (sufficiently high magnetic field), then the distribution of partial widths $\Gamma_{\lambda}$ should be a $\chi_{\nu}^{2}$ distribution with the degrees of freedom $\nu=1$ and $\nu=2$ respectively 2 . This distribution should be universal in the chaotic regime, i.e. two different shapes both of which generate chaotic classical dynamics should have the same distribution of level widths (even though the individual levels are quite different). However if the system approaches integrability then non-universal distributions differing from $\chi^{2}$ should arise. Precisely this behavior is confirmed by our numerical calculations of the partial width distribution for the conformal billiard model as seen in Fig. 12, where the fully chaotic GOE models (c) and (d) fits a $\chi_{\nu=1}^{2}$ distribution, the fully chaotic GUE models (e) and (f) fits a $\chi_{\nu=2}^{2}$ distribution, while neither the regular model (a) nor the soft-chaotic model (b) fits a $\chi_{\nu}^{2}$ distribution. A more extensive demonstration of the universality in the chaotic regime is seen in Fig. 13, where we plot for each value of the shape parameter $\delta$ in the deformation sequence of Fig. $2 \mathrm{~b}$ the value of $\nu$ yielding the best $\chi^{2}$ fit of a $\chi_{\nu}^{2}$ distribution to the numerically calculated histograms both without and with an AB-flux. It is seen that in all cases the value of $\nu$ fluctuates around the expected line $\nu=1$ (zero AB-flux) and $\nu=2$ (non-zero AB-flux).

Having confirmed that random-matrix theory works well in the chaotic regime, one can derive 12 from Eq. (43) the probability density $\mathcal{P}_{\nu}(\alpha)$ where $\nu=2$ for the orthogonal case and $\nu=4$ for the unitary case. One finds

$$
\begin{aligned}
& \mathcal{P}_{2}(\alpha)=\sqrt{2 / \pi \alpha} e^{-2 \alpha} \\
& \mathcal{P}_{4}(\alpha)=4 \alpha\left[K_{0}(2 \alpha)+K_{1}(2 \alpha)\right] e^{-2 \alpha},
\end{aligned}
$$


where $K_{n}$ are the modified Bessel functions of the second kind. $\mathcal{P}_{2}$ and $\mathcal{P}_{4}$ are plotted in Fig. 14 where they are compared to numerical data obtained by evaluating $\alpha$ in Eq. (43) for the 300 lowest wave functions of the conformal model for NBC in both the GOE and the GUE case. The time-reversal symmetry-breaking needed to study the GUE case is achieved by adding an AB-flux of one quarter of a flux quantum. In contrast to the results in Ref. 12 we find excellent agreement between random-matrix theory and numerical calculation in both the GOE and the GUE case. Note the substantial suppression of small peak amplitudes caused by breaking time-reversal symmetry. This reduces substantially the variance of $\alpha$, from Eqs. (44) and (45) one finds $\Delta \alpha_{4}^{2} / \Delta \alpha_{2}^{2}=32 / 45 \approx 0.71$.

The effect of a TR-symmetry breaking magnetic field on the distribution and its moments provides perhaps the simplest experimental test of our theory. However if the suppression of the amplitude fluctuations due to time-reversal symmetry breaking is to be cleanly observable then the magnetic field necessary to induce the GOE-GUE transition must be small compared to that needed for Landau level formation. Landau level formation strongly suppresses the fluctuations 41 ; the classical analogue of this effect is the suppression of chaos by the formation of stable skipping orbits2 . We estimate the magnetic field scale for TRsymmetry breaking by adapting an argument first put forward (to our knowledge) by Berry and Robnik29. TR-symmetry is broken first not by the dynamic effect of the field but by its effect on the phase of the wavefunctions (essentially the Aharonov-Bohm effect). Therefore in estimating the TR-symmetry breaking scale we may neglect the dynamic effect of the field entirely. Gutzwiller's trace formulat implies that structure in the spectrum on the scale of the level spacing $\Delta \varepsilon$ arises from periodic orbits of period $T \approx \hbar / \Delta \varepsilon$. A magnetic field will change the action (in units of $\hbar$ ) of such orbits by $B A_{T} /(h / e)$, where $A_{T}$ is the area enclosed by the periodic orbit of period $T$ in the chaotic case. The time-reversed orbit will of course enclose area $-A_{T}$ and their relative phases will be shifted by order unity when $B A_{T} \approx(h / e)$, breaking TR-symmetry. Thus the critical field $B_{c}$ for TR-symmetry breaking is given by $B_{c} \sim(h / e) / A_{T}$ and one need only estimate $A_{T}$. Berry and Robnik 29 treated the case of an A-B flux as above and then evaluated the mean-squared winding number for such orbits in the chaotic limit. Their results can be extrapolated to a uniform field simply by assuming a typical (positive or negative) area of order $A$ (the area of the dot) is enclosed with each circuit. With this modest assumption the TR-symmetry breaking flux

$$
\Phi_{c}=B_{c} A \sim\left[\Delta \varepsilon \sqrt{A} / \hbar v_{f}\right]^{1 / 2}(h / e) .
$$

Although in the experiments the dot is not isolated as assumed in this argument, the condition $\bar{\Gamma} \ll \Delta \varepsilon$ insures that electrons remain in the dot long enough for the argument to still apply. The ratio of this field to the field at which the cyclotron radius becomes of order the radius of the dot scales like $N^{-3 / 4}$, where $\mathrm{N}$ is the number of electrons; so in dots containing a few hundred electrons TR-symmetry breaking occurs at a field one to two orders of magnitude smaller than that needed for edge-state formation. In the experimental systems of interest this corresponds to a field of order a few times $10 \mathrm{mT}$. Thus the statistical effect of time-reversal symmetry breaking predicted by our theory should be observable experimentally 


\section{OPTIMAL EXPERIMENTAL SETUP}

To obtain the most direct experimental verification of the theoretical results presented here and in earlier work it is desirable to fabricate a quantum dot with a variable shape. In current experimental systems only a few tens of CB peaks are measured in a given dot and these are superimposed on a significant background which complicates the comparison of the height of widely-separated peaks. Thus obtaining reasonable statistics (e.g. roughly one hundred peak amplitudes) is very difficult. It is possible to use the magnetic field itself as an external control parameter causing peak amplitude fluctuations 14 , but this only allows one to collect statistics for the GUE case. Therefore we suggest an optimal heterostructure for tests of RMT statistics would consist of a dot formed by multiple teeth-like gates (see Fig. 15). By changing the voltages slightly on the inner gates of this structure it should be possible to realize a whole range of shapes without affecting the region near the quantum point contacts defining the tunnel barriers to the surrounding two-dimensional electon gas. It would then be possible to follow a given peak as a function of shape and collect statistics without the complication of background variation, both in the presence and absence of a magnetic field. Since the magnetic field would not be the control parameter it would also be possible to map out the GOE to GUE transition. This concept is illustrated by the calculations for the deformed billiard shown in Fig. 16 where we plot the peak amplitude $\alpha$ for two given levels as function of the shape parameter $\delta$. A particularly simple quantity to extract from such experiments is the correlation function for the peak amplitude as the shape is varied. Although we are not able to make quantitative estimates at this point our results suggest that for dots containing a few hundred electrons (as in current experiments on micron-size devices) quite small voltages would be needed to rearrange the wavefunctions of the highest levels and hence decorrelate the peak amplitudes. In the single-level regime $(k T<\Delta \varepsilon)$ the theory predicts that the voltage scale should be independent of temperature; a prediction which could be experimentally tested and (if confirmed) would provide support for the basic model.

\section{DISCUSSION AND CONCLUSION}

In this paper we have studied the statistical properties of deformable billiards in the mixed and fully chaotic limits, with applications suggested to quantum dot systems. We have presented numerical results based on the continuous family of conformal quantum billiards introduced by Robnik for which efficient numerical solution of the Schrödinger equation is possible and for which a rather complete characterization of the classical mechanics exists, including the existence of a KAM transition.

This model system has provided us with a unifying framework within which we discussed several aspects of quantum chaos in billiards. Our calculations demonstrate the universal behavior of the system once chaos is sufficiently strong (hard chaos) in which case randommatrix theory describes the quantum statistical properties independent of the details of the billiard geometry or the boundary conditions on the quantum problem. The calculations also demonstrate that in the mixed regime (soft chaos) the behavior is not universal and the random-matrix ensembles do not describe the system. We have also verified the theoreti- 
cal results obtained in the literature for the parametric energy level correlations of isolated quantum dots and the statistics of Coulomb blockade conductance peaks based on the assumption that RMT or the supersymmetric $\sigma$ model applies to chaotic billiards thereby adding credibility to these assumptions. Finally, we have proposed an experimental setup which is particularly well suited for tests of the theoretical predictions in this work.

\section{ACKNOWLEDGEMENTS}

We would like to thank R. Torsten Clay for taking part in the preliminary calculations of the classical properties in the billiards and B. Simons for helpful discussions and for allowing us to use the numerical correlation functions for disordered systems displayed in Fig. 11. We thank Marko Robnik for helpful discussions as well. This work is partially supported by ARO grant DAAH04-93-G-0009. H.B. is grateful for support by grant no. 11-0271 from the Danish Natural Science Research Council. 


\section{REFERENCES}

${ }^{1}$ For review of the physics of semiconducting mesoscopic devices see C.W.J. Beenakker and H. van Houten, Solid State Phys. 44, 1 (1991). Reviews of some aspects of quantum chaos in nanostructures can be found in Chaos 3, (1993), articles by Marcus et al, Lin et al. and Baranger et al.

${ }^{2}$ B.L Altshuler and B.I. Shklovskii, Zh. Eksp. Teor. Fiz . 91, 220 (1986) [Sov. Phys.-JETP 64, 127 (1986)].

${ }^{3}$ N. Argaman, Y. Imry and U. Smilansky, Phys. Rev. B 47, 4440 (1993).

${ }^{4}$ For a recent review see M. C. Gutzwiller, Chaos in Classical and Quantum Mechanics, Springer Verlag (New York, 1990)

${ }^{5}$ O. Bohigas, M.-J. Giannoni, and C. Schmit, Phys. Rev. Lett. 52, 1 (1984).

${ }^{6}$ R. A. Jalabert, H. U. Baranger, and A. D. Stone, Phys. Rev. Lett. 65, 2442 (1990).

${ }^{7}$ C. M. Marcus, A. J. Rimberg, R. M. Westervelt, P.F. Hopkins, and A. C. Gossard, Phys. Rev. Lett. 69, 506 (1992).

${ }^{8}$ M. W. Keller, O. Millo, A. Mittal, D. E. Prober, and R. N. Sachs, Surf. Sci. 305, 501 (1994).

${ }^{9}$ H. U. Baranger, R. A. Jalabert, and A. D. Stone, Phys. Rev. Lett. 70, 3876 (1993).

${ }^{10}$ U. Meirav, M. A. Kastner and S. J. Wind, Phys. Rev. Lett. 65, 771 (1990).

${ }^{11}$ L. P. Kouwenhoven et al., Z. Phys. B 85, 367 (1991).

${ }^{12}$ R. A. Jalabert, A. D. Stone, and Y. Alhassid, Phys. Rev. Lett. 68, 3468 (1992).

${ }^{13}$ A.D. Stone, R.A. Jalabert, and Y. Alhassid, in: Springer Series in Solid-State Sciences, Vol. 109, Eds. H. Fukuyama and T. Ando (Springer, Berlin, 1992) p. 39.

${ }^{14}$ A. D. Stone and H. Bruus, Physica B 18943 (1993), and Surf. Sci. 305490 (1994).

${ }^{15}$ V. N. Prigodin, K. B. Efetov, and S. Iida, Phys. Rev. Lett. 711230 (1993).

${ }^{16}$ H.U. Baranger and P.A. Mello, unpublished.

${ }^{17}$ C.W.J. Beenakker, R.A. Jalabert and J.-L. Pichard, unpublished.

${ }^{18}$ M. L. Mehta, Random Matrices and the Statistical Theory of Energy Levels (Academic, New York, 1991) 2nd ed.

19 T. A. Brody, J. Flores, J. B. French, P. A. Mello, A. Pandey, and S. S. M. Wong, Rev. Mod. Phys. 53, 385 (1981).

${ }^{20}$ M. V. Berry, Proc. Roy. Soc. Lond. A 400, 229, (1985).

${ }^{21}$ R. Balian, Nuovo Cimento 57, 183 (1968).

${ }^{22}$ M. V. Berry and M. Tabor, Proc. R. Soc. Lond. A 356, 375 (1977).

${ }^{23}$ A. Szafer and B. L. Altshuler, Phys. Rev. Lett. 70, 587 (1993)

${ }^{24}$ B. D. Simons and B. L. Altshuler, Phys. Rev. Lett. 70, 4063 (1993) and Phys. Rev. B 48, 5422 (1993).

${ }^{25}$ U. Sivan et al. Europhys. Lett. 25, 605 (1994).

${ }^{26}$ L.E. Reichl, The Transition to Chaos (Springer Verlag, New York, 1992).

${ }^{27}$ M. Robnik, J. Phys. A 16, 3971 (1983).

${ }^{28}$ M. Robnik, J. Phys. A 17, 1049 (1983).

${ }^{29}$ M. V. Berry and M. Robnik, J. Phys. A 19, 649 (1986).

${ }^{30}$ A. Hayli, T. Dumont, J. Moulin-Ollagnier, and J.-M. Strelcyn, J. Phys. A 20, 3237 (1987).

${ }^{31}$ M. Robnik and M. V. Berry, J. Phys. A 19, 669 (1986).

${ }^{32}$ R. Markarian, Nonlinearity 6, 819 (1993) 
${ }^{33}$ M. V. Berry, Eur. J. Phys. 2, 91 (1981).

${ }^{34}$ G. Benettin and J.-M. Strelcyn, Phys. Rev. A 17773 (1978).

${ }^{35}$ J. Goldberg, U. Smilansky, M. V. Berry, W. Schweizer, G. Wunner, and G. Zeller, Nonlinearity 4, 1 (1991).

${ }^{36}$ H. P. Baltes and E. R. Hilf Spectra of Finite Systems (Wissenschaftsverlag, Bibliographisches Institut, Zürich, 1976).

${ }^{37}$ H. Feshbach, Phys. Rev. 65, 307 (1944), and Chap. 9 in P. M. Morse and H. Feshbach, Methods of theoretical physics (McGraw-Hill, New York, 1953).

${ }^{38}$ Y. Meir, N. Wingreen and P. A. Lee, Phys. Rev. Lett. 66, 3048 (1991).

39 A.M. Lane and R.G. Thomas, Rev. Mod. Phys. 30, 257 (1958).

${ }^{40}$ C. W. J. Beenakker, Phys. Rev. B 44, 1646 (1991).

${ }^{41}$ R.A. Jalabert and A.D. Stone, unpublished.

${ }^{42}$ M. Robnik and M. V. Berry, J. Phys. A 18, 1361 (1985). 


\section{FIGURES}

FIG. 1. The deformed billiard $\mathcal{D}$ is the image of the unit disk $\mathcal{C}$ under the mapping $w(z)$, which is conformal $\left(w^{\prime}(z) \neq 0, \forall z \in \mathcal{C}\right)$ and area preserving $\left(\int_{\mathcal{C}}\left|w^{\prime}(z)\right|^{2} d z=\pi\right)$.

FIG. 2. Two sequences, (a) and (b), of $\partial \mathcal{D}$ as a function of changing parameters. Sequence (a) was studied by Robnik27. Sequence (b) is studied in this paper. For the very last shape in each sequence $w(z)$ is in fact not conformal. In both of these extreme cases $w^{\prime}(z)=0$ just at the point $z=-1$.

FIG. 3. Four Poincaré surface of sections for the deformation sequence shown in Fig. 2a.

FIG. 4. The Kolmogorov entropy plotted versus the normalized deformation parameter $X$ for the two deformation sequences (a) and (b) in Fig. 2. In (a) $X=b$, and in (b) $X=\delta$.

FIG. 5. The level spacing histogram for the Africa billiard $b=c=0.2$ and $\delta=\pi / 3$ with an AB-flux of $\frac{1}{4} \Phi_{0}$ and with DBC. A $\chi^{2}$-test supports the hypothesis of GUE level statistics (the full line) $\left(P\left(\chi^{2}\right)=0.68\right)$, whereas the hypothesis of GOE level statistics (the dotted line) is rejected $\left(P\left(\chi^{2}\right)=0.0002\right)$.

FIG. 6. The $\chi^{2}$ test for the deformation sequence shown in Fig. $2 \mathrm{~b}$ in the case of DBC and with an AB-flux of $\frac{1}{4} \Phi_{0}$. The full line $(*)$ is the test for GUE statistics while the dashed line (o) is for GOE statistics. The horizontal line is the (logarithm of the) mean value $1 / 2$ around which a successful $\chi^{2}$-test ought to fluctuate. Note the cross-over between the two statistics near the symmetry points $\delta=0$ and $\pi$.

FIG. 7. The $\chi^{2}$ test for the deformation sequence shown in Fig. $2 \mathrm{~b}$ in the case of NBC and with an AB-flux of $\frac{1}{4} \Phi_{0}$. The full line $(*)$ is the test for GUE statistics while the dashed line (o) is for GOE statistics. The horizontal line is the (logarithm of the) mean value $1 / 2$ around which a successful $\chi^{2}$-test ought to fluctuate. Note the rather wide cross over region between the two statistics near the symmetry point $\delta=0$.

FIG. 8. A deformed billiard with two leads attached. The leads can either be completely open or they can connect to the billiard through a tunnel barrier. The natural boundary conditions in this case are GBC. The wavy lines inside the billiard is the nodal structure of an eigenstate as briefly discussed in Sec. VI.

FIG. 9. The $\chi^{2}$ test for the deformation sequence shown in Fig. 2 $\mathrm{b}$ in the case of GBC and zero AB-flux. The full line $(*)$ is the test for GUE statistics while the dashed line (o) is for GOE statistics. The horizontal line is the (logarithm of the) mean value $1 / 2$ around which a successful

$\chi^{2}$ test ought to fluctuate. 
FIG. 10. Part of the normalized energy spectrum (levels 225 to 274) of the deformation sequence Fig. 2 $2 \mathrm{~b}$ with an AB-flux of one quarter of a flux quantum and with DBC.

FIG. 11. In (a) is shown the numerical calculated energy level correlation function $c(x)$ for the fully chaotic deformation sequence Fig. 2pb both with $(*)$ and without (o) an AB-flux. The full (dotted) curve is the universal correlation function calculated with (with out) an AB-flux for disordered systems in Ref. 24. In (b) $c(x)$ is shown with zero AB-flux in both the soft chaos regime $(\triangle)$ of the first half of the deformation sequence Fig. 2a and the hard chaos regime (o) repeated from (a). Note the strong dip for small values of $x$ in the case of soft chaos.

FIG. 12. The distribution of the partial decay width, $\Gamma_{\lambda}$, for six different deformed quantum billiards. Shown are histograms representing our numerical results, $\chi_{\nu}^{2}$ distributions (full curves) serving as a guide for the eye $(\nu=1.0$ in (a)-(d) and 2.0 in (e)-(f)), and as inserts the particular shapes of the dot. In (a), (b), and (c) we used the simple quadratic map with $c=0$ and with $b=$ $0.00,0.14$, and 0.39 respectively. In (d), (e), and (f) we used the cubic map with $b=c=0.2$ and $\delta=\pi / 3,2 \pi / 3$, and $\pi / 3$. In (a) the model is integrable, in (b) it is nearly integrable, and in (c)-(f) it is fully chaotic. There is no AB-flux in (a)-(d) and one quarter of a flux quantum in (e) and (f).

FIG. 13. The values of $\nu$ obtained from $\chi^{2}$ fits of a $\chi_{\nu}^{2}$ distribution to the numerically calculated histograms of the distribution of the partial decay widths $\Gamma_{\lambda}$ for the entire deformation sequence in Fig. 2b with zero AB-flux (o) and with an AB-flux of $1 / 4 \Phi_{0}(*)$.

FIG. 14. Predicted distribution of peak amplitudes $\alpha$ in the presence (a) and in the absence (b) of time-reversal symmetry, compared to the numerically generated amplitude distribution obtained with the shape parameters $b=c=0.2$ and $\delta=\pi / 3$.

FIG. 15. A top view of a gated heterostructure. The gate is splitted into twelwe teeths. The two pairs at the ends form quantum point contacts leading to the surrounding two-dimensional electron gas. The remaining eight teeths define the shape of the quantum dot. In situation (a) all the interior gates have the same voltage. In (b) one gate has a slightly higher voltage and another slightly lower thereby deforming the dot while maintaining its area. The regions close to the quantum point contacts are essentially unaffected by this action.

FIG. 16. The variation in the peak amplitude $\alpha$ for two particular levels as a function of shape deformation $\delta$. 